\title{
Optical tomography of the aurora and EISCAT
}

\author{
H. U. Frey ${ }^{1 *}$, S. Frey ${ }^{2 * *}$, B. S. Lanchester ${ }^{3}$, M. Kosch ${ }^{4}$ \\ ${ }^{1}$ Space Sciences Laboratory, University of California, Berkeley CA 94720-7450, USA \\ ${ }^{2}$ Max-Planck-Institut für Extraterrestrische Physik, D-85740 Garching, Germany \\ ${ }^{3}$ University of Southampton, Department of Physics, Southampton, SO17 1BJ, UK \\ ${ }^{4}$ Max-Planck-Institut für Aeronomie, D-37189 Katlenburg-Lindau, Germany
}

Received: 17 November 1997 / Revised: 14 April 1998 / Accepted: 17 April 1998

\begin{abstract}
Tomographic reconstruction of the threedimensional auroral arc emission is used to obtain vertical and horizontal distributions of the optical auroral emission. Under the given experimental conditions with a very limited angular range and a small number of observers, algebraic reconstruction methods generally yield better results than transform techniques. Different algebraic reconstruction methods are tested with an auroral arc model and the best results are obtained with an iterative least-square method adapted from emission-computed tomography. The observation geometry used during a campaign in Norway in 1995 is tested with the arc model and root-mean-square errors, to be expected under the given geometrical conditions, are calculated. Although optimum geometry was not used, root-mean-square errors of less than $2 \%$ for the images and of the order of $30 \%$ for the distribution could be obtained. The method is applied to images from real observations. The correspondence of original pictures and projections of the reconstructed volume is discussed, and emission profiles along magnetic field lines through the three-dimensionally reconstructed arc are calibrated into electron density profiles with additional EISCAT measurements. Including a background profile and the temporal changes of the electron density due to recombination, good agreement can be obtained between measured profiles and the time-sequence of calculated profiles. These profiles are used to estimate the conductivity distribution in the vicinity of the EISCAT site. While the radar can only probe the ionosphere along the radar beam, the three-dimensional tomography enables conductivity estimates in a large area around the radar site.
\end{abstract}

Key words. Tomography $\cdot$ Aurora .

EISCAT $\cdot$ Ionosphere $\cdot$ Conductivity

\footnotetext{
*Former address: MPE Garching

${ }^{* *}$ Present address: Space Sciences Laboratory, UC Berkeley

Correspondence to: H.U. Frey, e-mail: hfrey@ssl.berkeley.edu
}

\section{Introduction}

The aurora is the optical signature of acceleration processes of electrons and protons in magnetospheric regions at several thousand kilometers' altitude. The collision of energetic electrons with ionospheric atoms and molecules creates auroral arcs via the optical emission during de-excitation and increases the ionospheric conductivity by the creation of secondary charged particles. The ionospheric conductivity is an important parameter for the investigation of energy dissipation from field-aligned currents and of ionospheric currents flowing in the polar ionosphere. The vertical optical emission and conductivity profiles strongly depend on the flux and energy spectrum of precipitating electrons. Measured profiles can be used together with models of the atmosphere to prove theoretical models of electron acceleration in the magnetosphere and to determine temporal and local changes of ionospheric properties.

Rocket and satellite measurements provided much information about plasma properties and acceleration processes inside or near the acceleration region in the magnetospheric-ionospheric system. However, the coincidence of their periodical pass with any auroral event is accidental. Ground-based optical and radar observations were always used as an additional source of information (Omholt, 1971; Röttger, 1991). The advantages of ground-based optical observations are the large field of view, high spatial and temporal resolution, permanent operation and low costs. Furthermore, cameras can easily be transported to other sites. Radar measurements use large immobile equipment and in the case of incoherent-scatter radars, only measurements along one line may be performed. The disadvantage of large-angle scanning measurements is the long time it takes for a complete scan when compared to the fastchanging ionospheric processes. 
With regards to our interest in the spatial distribution of auroral emission, quantitative tomographic reconstruction (Natterer, 1986) may recover the volume emission in physical coordinates. The goals are line profiles comparable with emission profiles calculated from model atmospheres, precipitation fluxes, and the quantitative determination of temporal changes of auroral emission.

Ionospheric tomography reconstructs the distribution of the electron density in an altitude-latitude plane from measurements of the total ionospheric electron content determined from satellite beacon or GPS measurements (Pryse and Kersley, 1992; Pakula et al., 1995; Raymund, 1995). These measurements, however, assume spatial homogeneity because the receiving stations sometimes do not exactly lie in one plane with the satellite orbit and therefore, the rays do not really intersect. Furthermore, temporal stationarity is a fundamental condition during the $\approx 20$-min horizon-tohorizon pass of the satellite (Walker et al., 1996). Thus, short temporal fluctuations cannot be studied with this method, and satellite passes need not necessarily coincide with interesting events. Nevertheless, this method achieved highly desired gross-scale results in large polar regions and the EISCAT radar provided fundamental independent data used for the verification of ionospheric reconstructions (Markkanen et al., 1995; Walker et al., 1996; Nygrén et al., 1996; Jakowski et al., 1996).

Photometer scanners and all-sky observations can be used in a similar way to obtain two-dimensional auroral volume emission reconstructions in an altitude-latitude plane (Aso et al., 1990; Vallance Jones et al., 1991; Doe et al., 1997). However, 2D images of the aurora contain information for a 3D reconstruction of the auroral volume emission rates (Gustavsson, 1992). As in ionospheric tomography, auroral tomography with groundbased optical instruments is restricted to a small number of cameras, sometimes with different properties. Generally, these instruments are located on the Earth's surface and therefore have a limited angular range for observations. Proper reconstruction methods have to account for these limitations.

Based on previous studies with the extreme case of a 3D reconstruction from only two stereoscopic images (Frey et al., 1996a), and with the investigation of different observation geometries and an increasing number of observers (Frey et al., 1996b), the aim of this paper is to compare the results of different reconstruction techniques to determine the reconstruction quality for a commonly used observation geometry. This comparison is made with an array of cameras observing a common volume through which the auroral arc moves. It was shown (Frey et al., 1996b) that a fundamental improvement of reconstruction from angular range limited observations can only be obtained by calibration with additional measured or determined quantities. As in the case of ionospheric radio tomography the EISCAT radar is used as the additional source of information. However, in contrast to the radio tomography we will use another approach by calibrating the reconstruction with plasma density measurements by the
EISCAT radar. Using this approach we are able to obtain a large-scale conductivity distribution around the EISCAT site in high temporal resolution and to extract altitude profiles even in those areas that are not probed by the radar beam.

\section{Instrumentation and image processing}

During a coordinated observation in January 1995, three CCD cameras of the Max-Planck-Institute for Extraterrestrial Physics (Frey et al., 1996c) and two of the Max-Planck-Institute for Aeronomy were operated in the vicinity of the EISCAT radar site (Fig. 1). The main camera parameters are summarized in Table 1.

Except for the all-sky camera in Skibotn, all cameras were pointed to $110 \mathrm{~km}$ altitude on the magnetic field line through the EISCAT site. All cameras used GPS time for exact timing and were operated in real-time imaging with 25 frames per second.

At the same time the EISCAT UHF radar was measuring in the PULSE program, which is designed to measure rapidly varying auroral events with a 0.2 -s time resolution. Here we use power profile measurements between 75 and $145 \mathrm{~km}$ altitude in time-steps of 1-s time resolution.

The result of tomographic reconstructions strongly depends on the quality of the input images. Real images taken with cameras are subject to geometry, sensitivity, background, and noise distortions. Furthermore, extraction of real coordinates from images needs to consider the projection of the celestial sphere to the plane of the CCD chip. The image processing procedures we used for noise reduction, geometry, background, and nonuniformity correction before reconstruction were explained in Frey et al. (1996a).

\section{Method}

Auroral arcs are manifold in shape, color, and dynamics. Therefore a suitable tomographic inversion technique may be applied to the wide variety of aurora without the requirement of any prescribed model but with the useful incorporation of a priori information about the geometry and the knowledge of the nonnegativity of auroral arc emission.

The properties of the aurora equal those of objects analyzed in emission-computed tomography (Budinger et al., 1979), with the projection or image $p_{i}(m, n)$ of a

Table 1. Parameters of the cameras run near Tromsø. FOV is the field of view

\begin{tabular}{llc}
\hline No. & position & FOV \\
\hline 1 & Ramfjord; $19.220^{\circ} \mathrm{E}, 69.534^{\circ} \mathrm{N}$ & $36^{\circ} \times 64^{\circ}$ \\
2 & Laksvatn; $19.413^{\circ} \mathrm{E}, 69.335^{\circ} \mathrm{N}$ & $36^{\circ} \times 64^{\circ}$ \\
3 & Skibotn; $20.36^{\circ} \mathrm{E}, 69.35^{\circ} \mathrm{N}$ & $170^{\circ} \times 170^{\circ}$ \\
4 & Ramfjord; $19.220^{\circ} \mathrm{E}, 69.534^{\circ} \mathrm{N}$ & $31^{\circ} \times 16^{\circ}$ \\
5 & Skibotn; $20.36^{\circ} \mathrm{E}, 69.35^{\circ} \mathrm{N}$ & $30^{\circ} \times 22^{\circ}$ \\
\hline
\end{tabular}


3D distribution $f(\mathbf{r})$ to the center at $\mathbf{r}=0$ described by the integral

$p_{i}(m, n)=C \int_{0}^{\infty} f(\mathbf{r}) \exp \left\{-\int_{s=0}^{s=r} \mu(s) d s\right\} \mathrm{d} \mathbf{r}$.

In the discrete case, the volume is divided into small cubes (voxel), with the value in each voxel $(x, y, z)$ representing the mean of the volume emission rate $f(x, y, z)$. Then, the integral is transformed into a sum with $h_{x y z}^{m n}$ describing the contribution of the voxel to the cone region subtended by the pixel $(m, n)$ for the observer $i$.

$p_{i}(m, n) \approx C^{\prime} \sum_{x, y, z} h_{x y z}^{m n} f(x, y, z)$.

The parameter $C$ contains the camera and lens parameters like spectral sensitivity, solid angle for the observation, filter and lens transmission, etc. The atmospheric attenuation is described by the parameter $\mu(s)$, and has to be taken into consideration if groundbased and space-based observations are combined. Because we only use ground-based observations, a constant attenuation can be included into a new constant parameter $C^{\prime}$.

The goal of tomographic reconstruction is to solve this set of linear equations. Various methods of 3D reconstruction from many $2 \mathrm{D}$ views have been developed and tested to find an estimate $f^{\prime}$ of $f$ (for reviews, see, e.g., Budinger and Gullberg, 1974; Gordon and Herman, 1974; Budinger et al., 1979; Natterer, 1986; Verhoeven, 1993).

Images from our optical observations have to be characterized as discrete, noisy, and limited data. Algebraic reconstruction techniques (ART) assume by design a discrete data set, a discrete reconstruction set, and they reconstruct the sample values of the projections to an accuracy only dictated by density and geometry in an iterative updating scheme. Additionally, they are

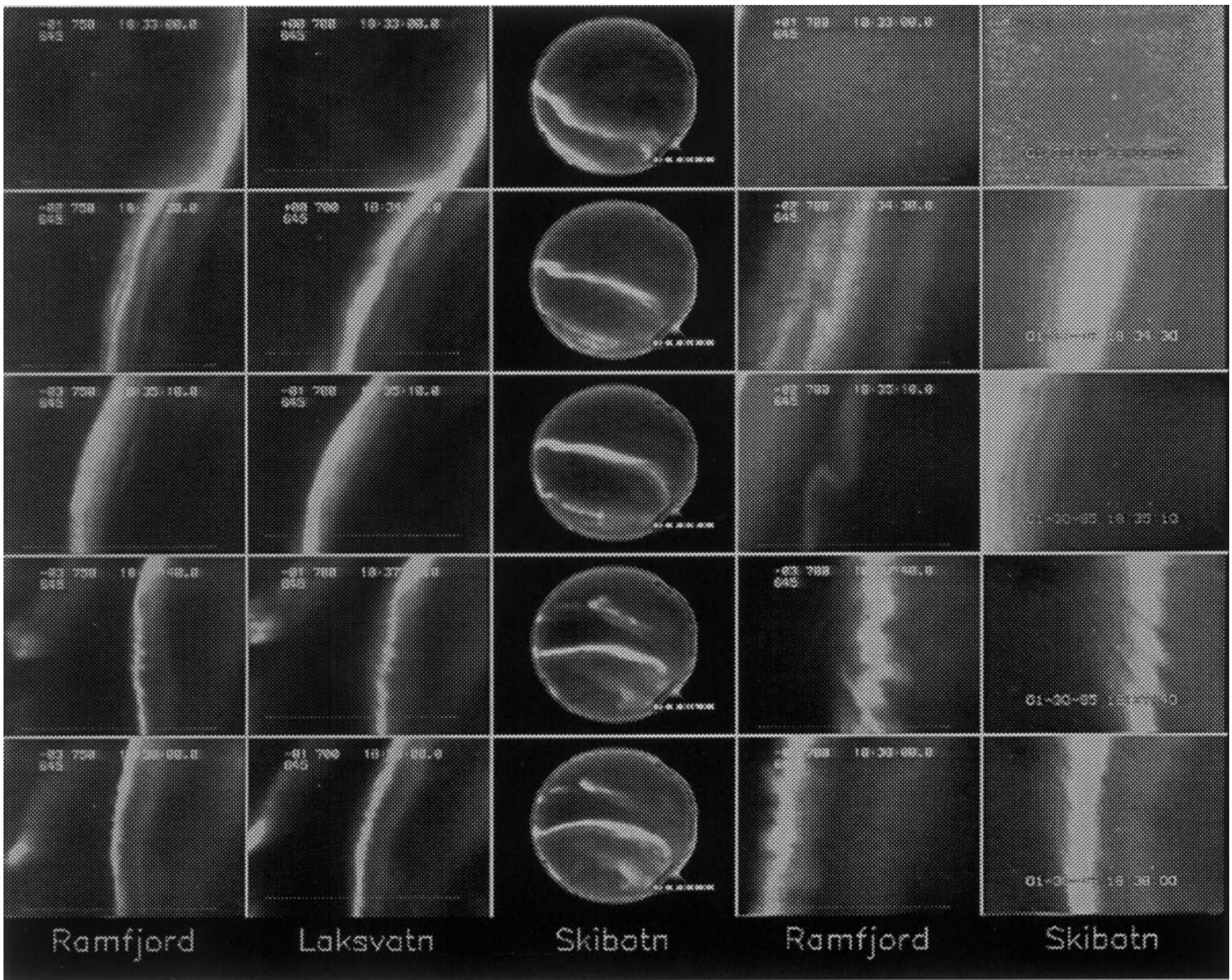

Fig. 1. Pictures of the aurora taken by the cameras in Norway on 30 January 1995. Long-pass red filters were used for the cameras at Ramfjord and Laksvatn. The camera parameters are given in Table 1. The times are from top to bottom 1833:00, 1834:30, 1835:10, 1837:40, and 1838:00 UT 
generally simple and flexible and permit the incorporation of a priori knowledge of the object's sign, support, and range. Depending on the number of observers, the size of the reconstruction volume, and errors in the data and discrete representation of the object, this set will either have many possible solutions or none at all.

Several types of ART differ in the way the corrective term is determined and applied to the object function while the iteration is always performed on a ray $l(r, \theta)$ by ray basis until the calculated projection $p_{i, l(r, \theta)}^{k}$ of the result of the last iteration step $k$ is as close as possible to the measured or original data $p_{i, l(r, \theta)}^{e}$ (Gilbert, 1972).

Mainly, we distinguish three types: additive ART (ART) with the nonnegative constraint

$f^{\prime k+1}(x, y, z)=f^{\prime k}(x, y, z)+b_{m n}^{x y z}\left(p_{i, l(r, \theta)}^{e}-p_{i, l(r, \theta)}^{k}\right) \geq 0 ;$

multiplicative ART (MART)

$f^{\prime k+1}(x, y, z)=f^{\prime k}(x, y, z) \cdot b_{m n}^{x y z} \frac{p_{i, l(r, \theta)}^{e}}{p_{i, l(r, \theta)}^{k}} ;$

simultaneous iterative ART (SIRT)

$f^{\prime k+1}(x, y, z)=f^{\prime k}(x, y, z) \cdot \frac{1}{I} \sum_{i} b_{m n}^{x y z} \frac{p_{i, l(r, \theta)}^{e}}{p_{i, l(r, \theta)}^{k}}$.

The weighting factor $b_{m n}^{x y z}$ is the inverse of $h_{x y z}^{m n}$.

To prevent the incorporation of a prescribed model (Aso et al., 1990) or a large data base of possible profiles (Walker et al., 1996), we reconstruct the first estimate with a modification of the simple back-projection (Budinger and Gullberg, 1974). The back-projection reconstructs the back-projected data $B(x, y, z)$ from a series of projections $p_{i}(m, n)$

$B(x, y, z)=\frac{T}{T^{\prime}} \cdot \sum_{i, m, n} b_{m n}^{x y z} p_{i}(m, n)$,

where for each element the contribution of each ray passing through that element is summed (Katsulai and Arimizu, 1985; Peyrin, 1985). The images are backprojected by filling one horizontal slice of the volume after the other. The quantitative correspondence is performed by a correction with the total density $T$ of the element and the total density $T^{\prime}$ of the complete array. This method is very fast but the agreement between the original and reconstructed object is only qualitative because too many simplifications are included. In our modification each cell is assigned with the minimum value of the back-projected data originating from the images of the observers $i$.

$f^{\prime}(x, y, z)=\min _{i}\left\{b_{m n}^{x y z} p_{i}(m, n)\right\}$.

This modification provides the advantage that a cell once set to a content of 0.0 due to the result of one of the projections cannot increase content.

During all iteration steps the ray sums calculated in the $k$-th iteration $p_{i, l(r, \theta)}^{k}$ are compared with the ray sums $p_{i, l(r, \theta)}^{e}$ measured along ray $l(r, \theta)$. These image ratios $p_{l(r, \theta)}^{e} / p_{l(r, \theta)}^{k}$ or differences $p_{l(r, \theta)}^{e}-p_{l(r, \theta)}^{k}$ are the input of a new back-projection. The content of the reconstructed volume elements are modified by multiplying or adding the content of all volume elements along a ray $l$ with the corresponding value of the back-projected data of the image ratios or differences due to each observer [MART, Eq. (4), or ART Eq. (3)] or with their mean [SIRT Eq. (5)]. These iterations are performed for all observers until a quality parameter $\lambda_{k}$ is minimized

$$
\begin{aligned}
& \frac{1}{I} \sum_{i} \sum_{x} \sum_{y} \sqrt{\left[p_{l(r, \theta)}^{e}(x, y, i)-p_{l(r, \theta)}^{k}(x, y, i)\right]^{2}} \\
& \quad=\lambda_{k} \cdot \sum_{i} \sum_{x} \sum_{y} p_{l(r, \theta)}^{e}(x, y, i) .
\end{aligned}
$$

This parameter is used to stop the iteration whenever the criterion is fulfilled that $\lambda_{k+1} \geq \lambda_{k}$. In contrast to Frey et al. (1996a) we introduced the factor $1 / I$ because otherwise, for an increasing number of observers $I$, the quality parameter would increase and counterfeit a worse reconstruction.

This parameter $\lambda_{k}$, however, is only relevant to the coincidence between the observed image and the reconstructed image. It does not guarantee the agreement between the reconstructed $f^{\prime}(x, y, z)$ and the original distribution $f(x, y, z)$ in the volume. As a quantitative comparison between the volume data a reconstruction parameter $\Lambda_{k}$ is defined

$$
\begin{aligned}
& \sum_{x} \sum_{y} \sum_{z} \sqrt{\left[f(x, y, z)-f^{\prime}(x, y, z)\right]^{2}} \\
& =\Lambda_{k} \cdot \sum_{x} \sum_{y} \sum_{z} f(x, y, z) .
\end{aligned}
$$

The reconstruction algorithms all use the perspective projection for imaging the reconstructed volume distribution. The complete procedure assumes a fixed Cartesian coordinate system for the description of the input parameters of all observers and a reference point within the object all cameras are looking at. The geometric calculations of the parameters $h_{x y z}^{m n}$ and $b_{m n}^{x y z}$ with the relative position between observer and object, the view direction, and the perspective (cone-beam geometry) are simplified using homogeneous coordinates (Foley and Van Dam, 1982).

\section{Results of theoretical modeling}

For tests of the reconstruction methods by means of the quality and reconstruction parameters and emission profiles along magnetic field lines, observations of a theoretical auroral arc model by three cameras were simulated with assumptions matching our real conditions.

A model of a Chapman-type auroral arc was calculated in geocentric magnetic coordinates within a volume of $61 \times 61 \times 61$ voxels corresponding to a distance of $110 \mathrm{~km}$ in each direction, with the following assumptions (Frey et al., 1996a). (1) The arc is extended in the geomagnetic east-west direction and the smooth 
shape was changed into an auroral arc-like shape by applying a sinusoidal modulation and a normally distributed random number process. (2) The symmetric latitudinal profile is a simplified Gaussian profile. (3) The altitude profile is an asymmetric, height-dependent, Chapman-type profile.

The altitude of peak brightness was chosen to be at $130 \mathrm{~km}$, and the Gaussian profile was calculated with a full width at half maximum (FWHM) of $4.0 \mathrm{~km}$. In Fig. 2 the arc is shown with the planes of $5 \%$ of maximum volume emission, together with the central magnetic field line. The observation of this arc was simulated for three cameras separated at locations corresponding to the observation geometry in Norway 1995.

Different observation geometries were tested in recent studies (Frey et al., 1996a, b) and the results will only briefly be summarized. The cameras have to have a minimum distance of $20 \mathrm{~km}$ from each other, but too large distances of more than $200 \mathrm{~km}$ result in poor spatial resolution and bigger differences in the reconstructed volume. Already two cameras in optimum
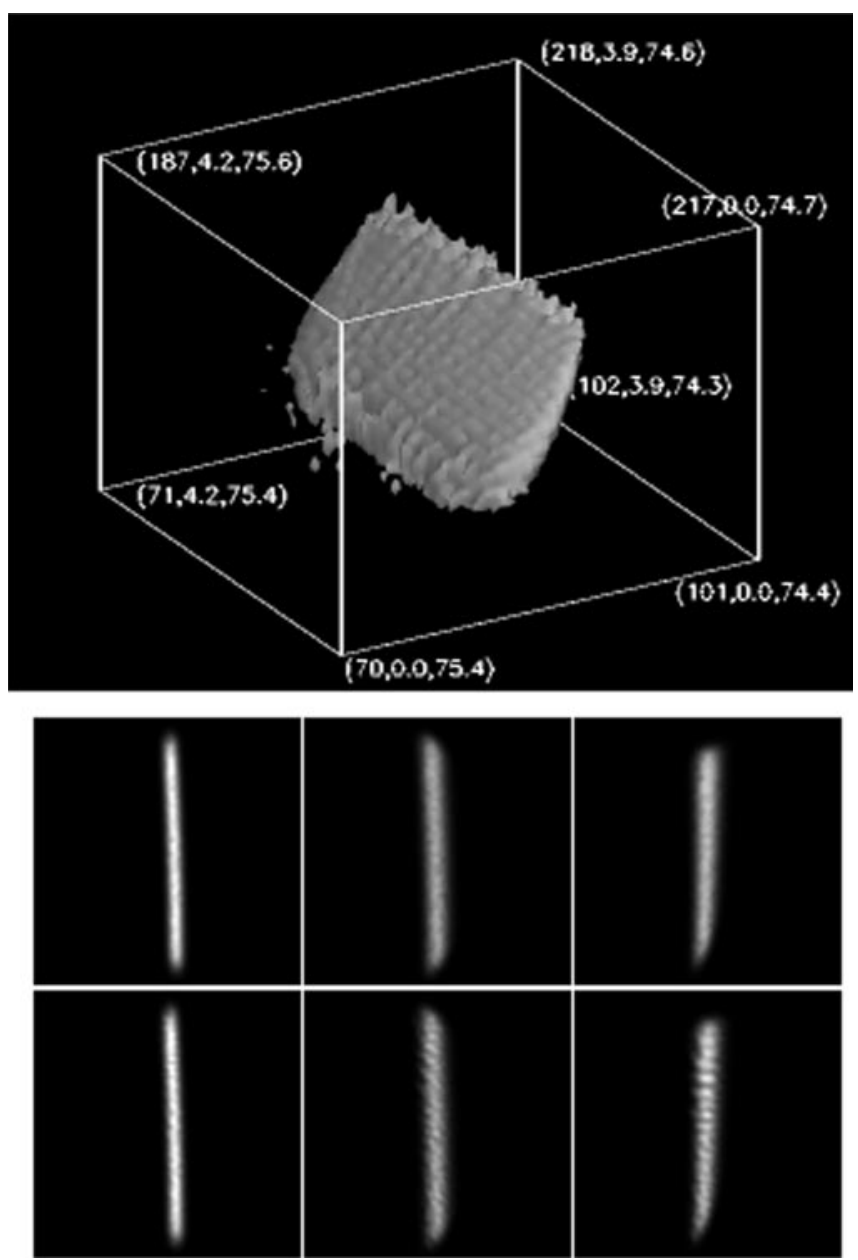

Fig. 2. Model of an auroral arc within a $61 \times 61 \times 61$ voxel volume. Each cube mark represents altitude, geocentric magnetic longitude, and geocentric magnetic latitude. The images correspond to images taken by cameras from three locations according to the observation geometry used in Norway, 1995 (lower images) and the images of the MART reconstructed arc are shown at the top observation geometry yield reasonable results, however, with between three and five cameras minimum quality and reconstruction parameters of $\lambda \approx 0.01$ and $\Lambda \approx 0.15$ can be obtained, respectively. The best results are obtained when the auroral arc is closer than $30 \mathrm{~km}$ to the magnetic zenith of one of the observers.

The camera locations for the Tromsø campaign were determined before the first results of theoretical modeling were obtained. The distance of $23 \mathrm{~km}$ between the cameras in Ramfjord and Laksvatn is too small in order to obtain the best results of reconstruction, which would be obtained at a distance of about $40 \mathrm{~km}$. However, in the following example the Tromsø geometry was used in order to determine the expected errors.

Figure 3 shows the quality and reconstruction parameters for an auroral arc that moved across the reconstruction volume around the EISCAT site from north to south. For this example the modified MART yields the best results. The problem with all methods is that there is no confirmed mathematical or physical reason to set particular voxel contents to zero, except for the modified MART where this is done at least for all the voxels along a ray from one of the observers which showed zero content in the images. Because the total intensity within the images has to correspond to the total volume emission of the model, some voxels at the outer surface of the arc contain intensity which is then missing in the center voxels of the arc. This is also most probably the reason why radio tomography tends to overestimate the electron density on the topside of the reconstruction (Walker et al., 1996) and to produce thicker layers (Nygrén et al., 1996).

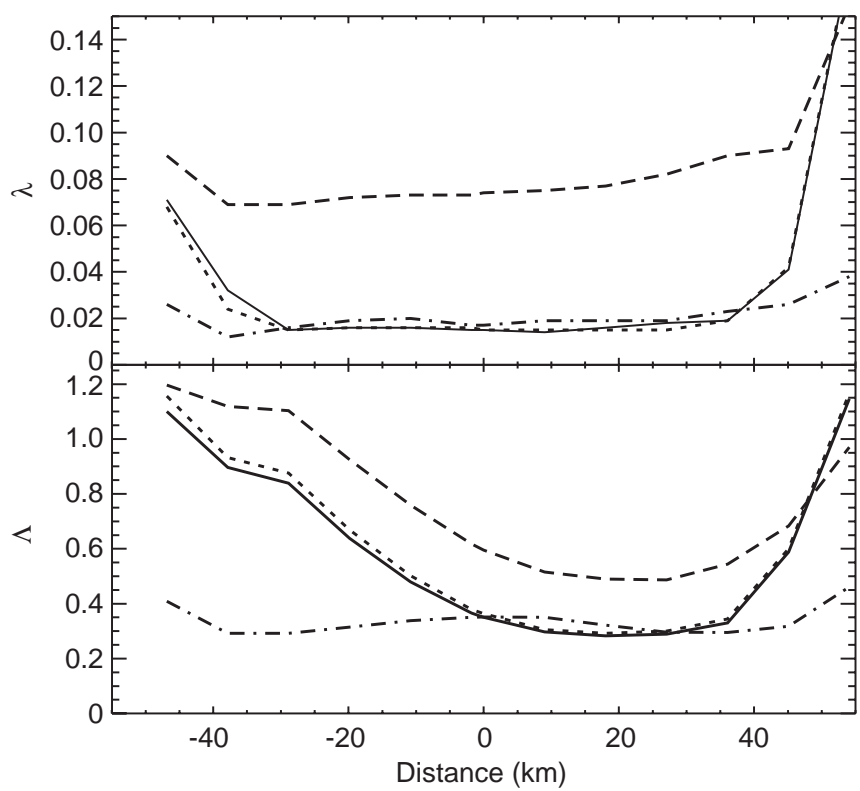

Fig. 3. Quality $\lambda$ and reconstruction $\Lambda$ parameters for the Troms $\varnothing$ observation geometry with three stations pointed to a fixed reference point and the auroral arc moving through the reconstruction volume from north to south. The results of ART (dashed line), SIRT (dotted line), and MART (solid line) are shown. The dash-dotted line connects the MART results with the first estimate taken from the reconstruction in the center of the volume 
In addition to the algebraic reconstruction methods Fig. 3 also shows an attempt to incorporate some a priori knowledge into the reconstruction method by regarding the images as a time-sequence of a moving object. The first reconstruction was performed with the arc at the magnetic zenith of the Ramfjord station. The result of this reconstruction was used as a first estimate for all other reconstructions. The relative location of the arc within the volume was determined and the reconstructed arc of the first estimate was shifted accordingly.

The result is that with this first estimate an even better reconstruction can be obtained if the arc has moved away from the observers. A voxel element within the reconstructed arc in the central position set to a content of 0.0 could never increase, but further, new voxel elements could be set to 0.0 due to the other parallax of the observers to the moved object. Though this method seems to be very promising for an arc far away from the magnetic zenith, it is only useful for objects which do not change shape during the time of investigation. For auroral arcs, which change shape within short time-periods, this approach may be tested, but may not be very useful in practice.

The similar trends of $\lambda$ and $\Lambda$ allow for the conclusion that both quantities are correlated and for our case of real image reconstruction we can stop the iteration whenever we reach the situation that $\lambda_{k+1} \geq \lambda_{k}$.

Noise always deteriorates the quality of real images. As a quantitative test two Gaussian noise components $n_{1}$ and $n_{2}$ were added to the content $I_{i}$ of the image pixels

$I_{i}^{*}=I_{i}+n_{1} \cdot I_{i}+n_{2}$

with increasing noise level and decreasing signal-tonoise ratio $S / N$ (Verhoeven, 1993)

$S / N=10 \log \left(\frac{\sum_{l=1}^{L} p_{l}^{2}}{\sum_{l=1}^{L} \Delta p_{l}^{2}}\right)$

where $\Delta p_{l}$ is the ray sum error caused by the noise (Fig. 4). Without noise reduction the results of the modified MART method already get much worse at signal-tonoise ratios of less than 50 . The nonnegative constraint improves the situation, but the median filter best improves to acceptable signal-to-noise ratios greater than 20 .

\section{Reconstruction of real observations}

Two parallel auroral arcs moved across the radar beam in a period of $7 \mathrm{~min}$ on 30 January 1995 after 18:33 UT (Fig. 1).

We chose a $61 \times 61 \times 61$ element reconstruction volume. Owing to reasons of calculation time, the central $303 \times 303$ pixel part of the images was resized to a final size of $101 \times 101$. Cameras 1,2 , and 3 were used for the reconstruction because cameras 4 and 5 were at the same locations and did not yield any more information.

The Ramfjord and Laksvatn images were taken with a long-pass blocking filter with a cutoff wavelength at

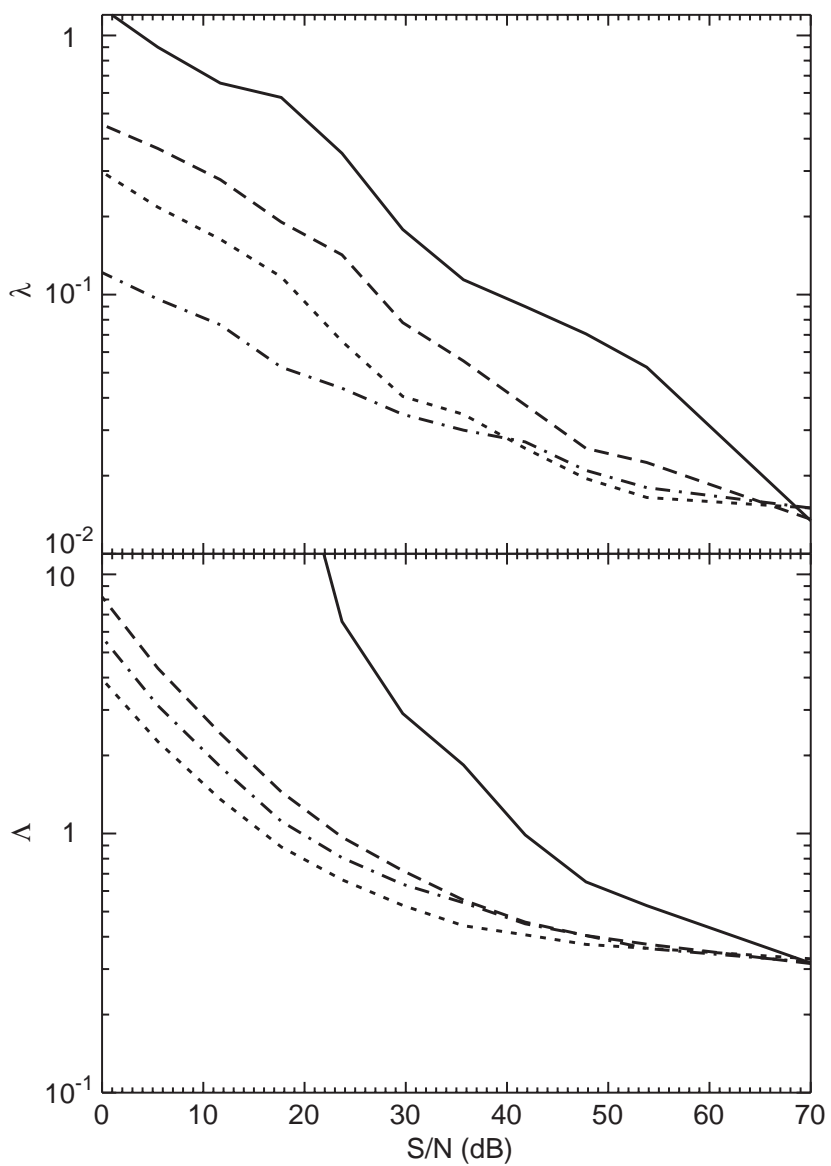

Fig. 4. Quality and reconstruction parameters for an increasing signalto-noise ratio in the original images and after application of different filters in the modified MART algorithm. The solid line represents MART without any filter, the dashed line MART with nonnegative constraint, the dotted line MART with median filter, and the dashdotted line MART with Fourier low-pass filter

$650 \mathrm{~nm}$, thus registering mostly the prompt emission of the first positive band of $\mathrm{N}_{2}$ and the Meinel band of $\mathrm{N}_{2}^{+}$. The all-sky camera at Skibotn used white light imaging and this introduces some additional uncertainty into the reconstruction process because generally one and the same wavelength band should be used for all cameras. We tried to minimize this uncertainty with additional weighting factors which were 1.0 for the Ramfjord and Laksvatn cameras, but 0.5 for the Skibotn camera. The procedure converges to a stable solution after two complete iterations with $\lambda_{2} \approx 0.7$.

The reconstruction sequence of $2.5 \mathrm{~min}$ (Fig. 5) reproduces the simulation results of Fig. 3. A much better result can be obtained when the arc was south of the radar beam, e.g. between the magnetic zenith of the Ramfjord and Laksvatn observers, when compared to positions north of all stations.

Auroral volume emission rates and electron stimulated ionization rates in the ionosphere are very closely correlated (Rees, 1963) and therefore, our optical profiles can be used to estimate the plasma density in the area around the EISCAT site. The reconstructed volume emission profile of an arc passing through the 


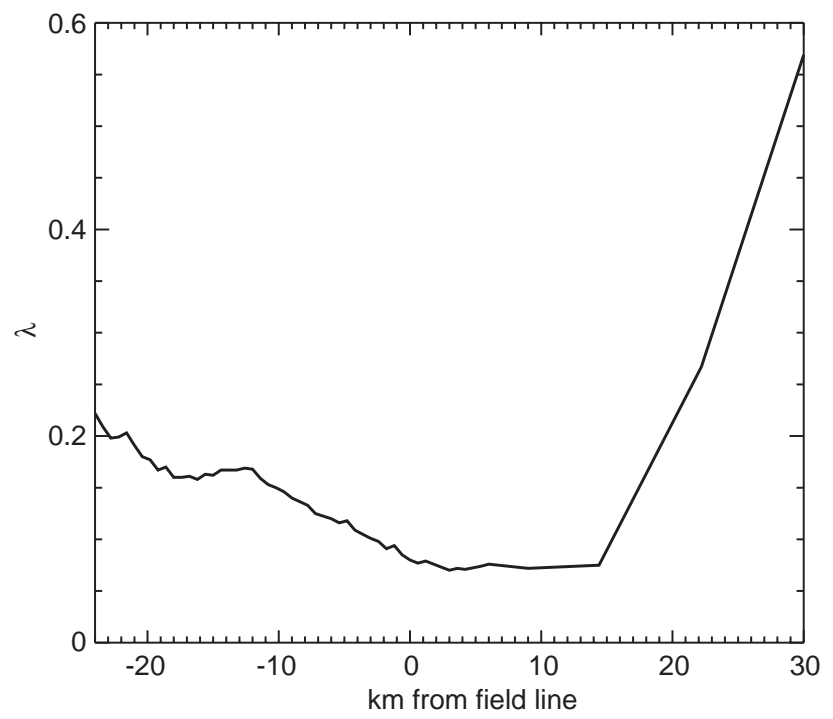

Fig. 5. Quality parameter $\lambda$ for the arc reconstruction from the Norway images for the time-period 1836:00-1838:30 UT on 30 January 1995 when the arc moved from north (positive distances between the arc center and the radar beam) to south through the EISCAT radar beam

radar beam at 1837:53 UT and the EISCAT-measured plasma density were used to calibrate the volume emission rate into electron density. Then the standard formula for the Pedersen

$\sigma_{p}=\left[\frac{v_{e n}}{v_{e n}^{2}+\omega_{g e}^{2}}+\frac{m_{e}}{m_{i}} \frac{v_{i n}}{v_{i n}^{2}+\omega_{g i}^{2}}\right] \frac{n_{e} e^{2}}{m_{e}}$

and the Hall conductivities

$\sigma_{h}=\left[\frac{\omega_{g e}}{v_{e n}^{2}+\omega_{g e}^{2}}-\frac{m_{e}}{m_{i}} \frac{\omega_{g i}}{v_{i n}^{2}+\omega_{g i}^{2}}\right] \frac{n_{e} e^{2}}{m_{e}}$

with the electron-neutral $v_{e n}$ and ion-neutral collision $v_{i n}$, and the electron gyro $\omega_{g e}$ frequencies were used (Schlegel, 1988). Further quantities are the electron density $n_{e}$, the unit charge $e$ and the electron mass $m_{e}$ and ion mass $m_{i}$. The MSIS-90 model atmosphere (Hedin, 1991) and a dipole magnetic field model were used to calculate the frequencies. The calculation of electron density profiles from the power profiles assumes equal electron and ion temperatures and a Debye length of the plasma much smaller than the radar wavelength. These assumptions tend to overestimate the real density in a sparse plasma, however the effect is small in our application with electron densities greater than $10^{11} \mathrm{~m}^{-3}$.

In our attempt to use the reconstructed emission profiles for estimating the ionospheric conductivities, we had to consider the relative motion of the field-aligned currents with reference to the ground-based observer. Furthermore, during the night without ionization by solar UV radiation, the electron densities in the $\mathrm{E}$ and $\mathrm{F}$ layers rapidly decrease until the new equilibrium of ionization by cosmic radiation and high-energetic solar particles is reached (Ratcliffe, 1972). Precipitating auroral electrons are an additional ionization source in the polar ionosphere, but after the disappearance of fieldaligned currents in a certain area the electron density decreases according to the continuity relation

$\frac{\mathrm{d} n_{e}}{\mathrm{~d} t}=q-\alpha n_{e}^{2}-\beta n_{e}$

with the ionization $q$, the recombination $\alpha$, and attachment $\beta$ coefficients, respectively. At lower altitudes the attachment can be neglected. Without new ionization $q=0$, Eq. (14) would result in the solution

$\frac{1}{n_{e}}-\frac{1}{n_{e o}}=\alpha t$.

The EISCAT data after the two arcs had passed the radar beam were used to fit recombination coefficients and 1.3-2.0 $10^{-13} \mathrm{~m}^{3} \mathrm{~s}^{-1}$ were obtained. Though these values are not very accurate if there was still some precipitation and ionization after the arcs had left the radar beam and though there was a height dependence of this quantity, we decided to use only one value of 2.0 $10^{-13} \mathrm{~m}^{3} \mathrm{~s}^{-1}$ for all heights. This value seems to be appropriate to the accuracy we need in our application.

In order to establish a background density profile within the reconstruction volume, a 15-s-integrated electron density measured long after the last obvious precipitation was used. The calibrated auroral emission profile was then used to calculate the additional electron density which in the following time-sequence was allowed to decrease according to Eq. (15).

The electron density profiles measured by the EISCAT radar are given in Fig. 6, together with the calibrated density profiles from the optical reconstructions. The background profile and the decreasing electron density from previous reconstructions are always added. The correspondence for the first times when the leading part of the arc came into the beam is not as good as for all later times. It may well be that low-energy electrons were the predominant source of ionization at the leading edge of the arc and did not excite as many of the first positive $\mathrm{N}_{2}$ and the Meinel $\mathrm{N}_{2}^{+}$ photons. The energy distribution of the incoming electrons can be estimated using the combination of an auroral model (Lanchester et al., 1994) and the measured electron density profiles. In this event the distribution is best fitted by a Maxwellian spectral distribution for most of the passage of the arc through the radar beam. The time-history of the peak energy of this distribution is shown in Fig. 7. It has a very low value of $500 \mathrm{eV}$ at the start, rising to $1.5 \mathrm{keV}$ at the peak, when the energy flux increased from only a few $\mathrm{mWm}^{-2}$ to $180 \mathrm{mWm}^{-2}$. For such a high energy flux it is unusual to find the peak energy so low.

The good correspondence between the measured and reconstructed/calibrated profiles allows the determination of the electron density profiles in the whole reconstruction volume, even away from the radar beam. These data were then used to calculate the heightintegrated Pedersen and Hall conductances in the area of $60 \times 60 \mathrm{~km}^{2}$ around the radar site (Fig. 8). The 


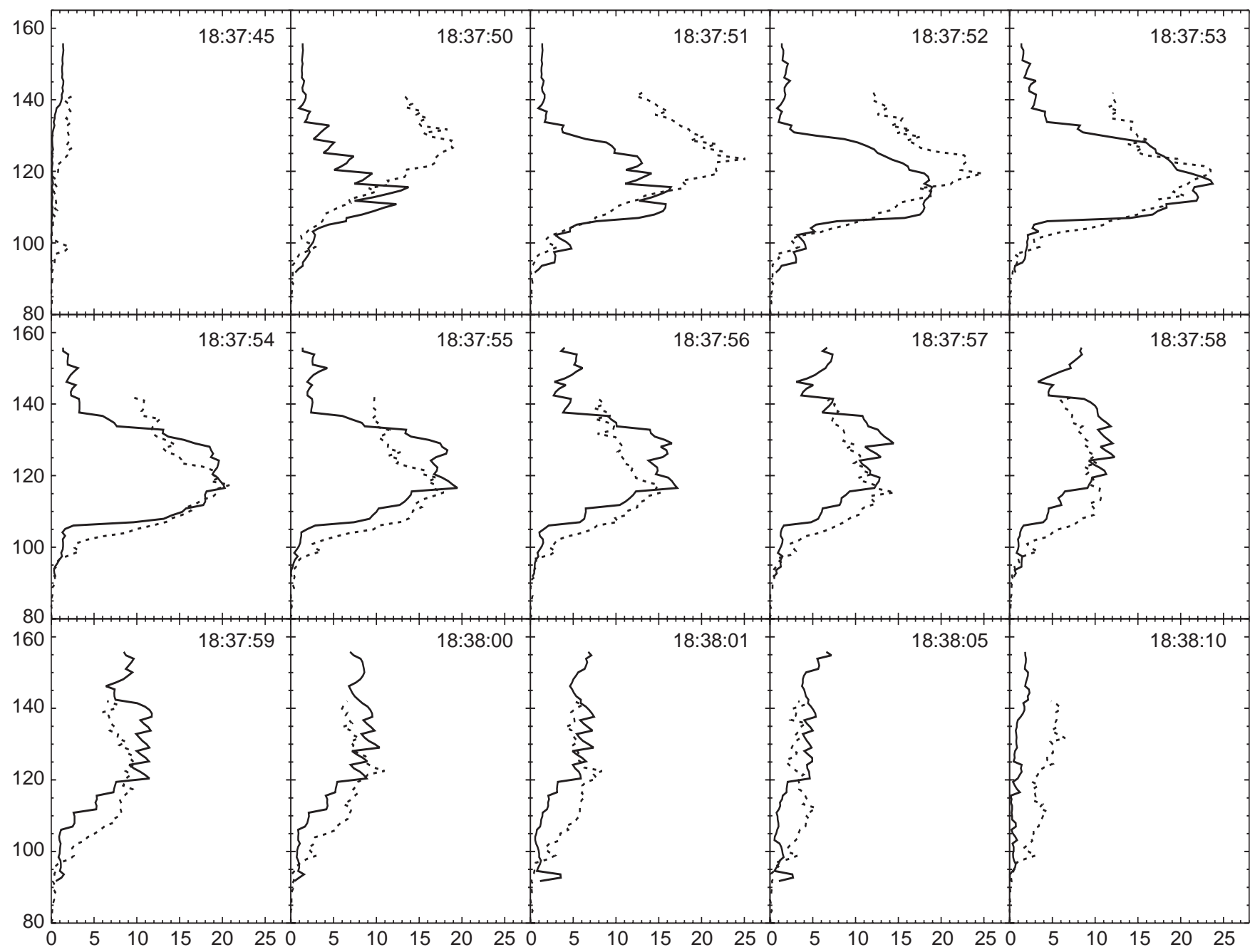

Fig. 6. Electron density profiles measured by the EISCAT PULSE program when the auroral arc crossed through the radar beam (dotted line) in units of $10^{11} \mathrm{~m}^{-3}$ and the calibrated reconstructed profiles (solid line). The left axis shows the altitude in $\mathrm{km}$. The profile at

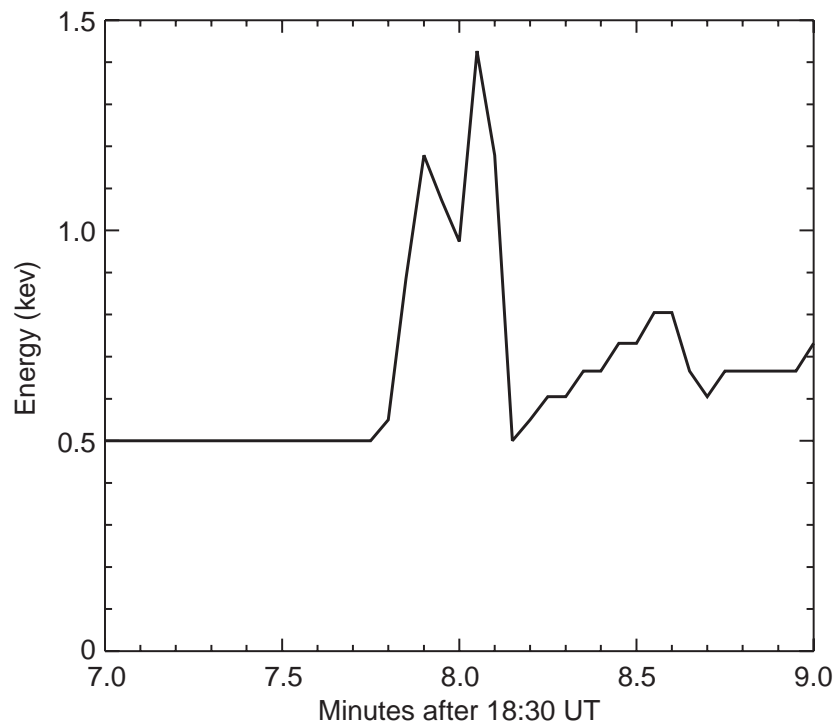

Fig. 7. Fitted Maxwellian peak energy of precipitating electrons for the time-period of arc passage through the EISCAT beam
1837:53 UT was used to calibrate the reconstructed optical volume emission to electron densities. A background density profile is always added and the previous profiles were allowed to decrease according to Eq. (15)

background profile created Hall and Pedersen conductances of $1.9 \mathrm{~S}$ and $2.5 \mathrm{~S}$, respectively, within the whole area. The observation in the prompt optical emission bands provides images of the instant electron precipitation and ionization. If we had not included the decreasing electron density from previous precipitation, we would have obtained only the volume emission profiles and the calibrated electron density profiles at the present position of the auroral arc. Our procedure provides a true determination of the electron density and conductances in those areas, where ionization occurred at previous times, but there is no optical emission at later times when the arc has moved away from the previous position.

Hall and Pedersen conductances of up to $110 \mathrm{~S}$ and $70 \mathrm{~S}$ within this time-interval are quite high (Lester et al., 1996), but not unusual for substorm expansion situations (Aikio and Kaila 1996). The IMAGE ground magnetometer system measured large negative bays in the $B_{x}$ component in all stations poleward of about $63.5^{\circ}$ magnetic latitude (data not shown). As we probe the 

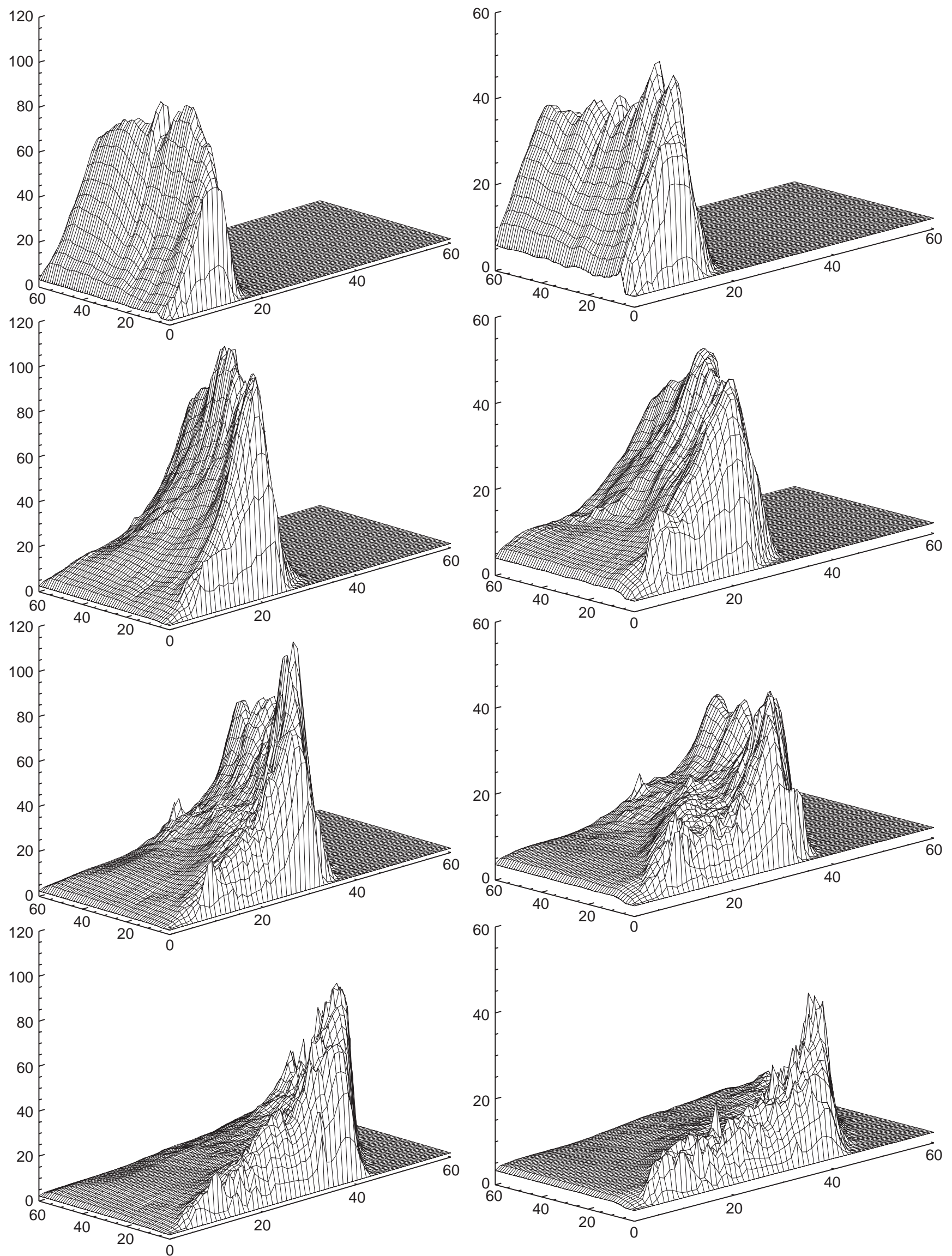
Fig. 8. Calculated height-integrated Hall (left) and Pedersen (right) conductances at 1836:30, 1837:53, 1838:10, and 1838:30 UT. The $x$ and $y$-axes correspond to the $60 \times 60 \mathrm{~km}^{2}$ area of reconstruction and the $z$-axis shows the conductances in units of $\mathrm{S}$

altitude region up to $160 \mathrm{~km}$ only and miss any conductivity in the higher $\mathrm{F}$ region, the true conductances are higher. The maximum Hall and Pedersen conductances along the EISCAT beam were $69 \mathrm{~S}$ and 64 $\mathrm{S}$, respectively. The higher maxima within the area represent the local differences of the ionization and excitation which also show up as different brightnesses within the optical images.

\section{Conclusions}

Three-dimensional reconstruction of auroral emission is possible with the presented iterative procedure. The method can be used to determine the minimum expected errors in a given observation geometry. Real observations suffer from different distortions, and even with suitable image processing the situation can not be very much improved.

For the test of the procedure, real images had to be used that were taken in an unfavorable observation geometry. Nevertheless, the auroral arc could be reconstructed and reasonable altitude profiles could be obtained. The calibration of the volume emission profiles with electron density profiles of the EISCAT radar provides good agreement if a background profile is used and if the optical observations are treated as a time-sequence of images of the instant ionization which is then allowed to decrease due to recombination.

The calibration of the reconstructed altitude profiles with the EISCAT electron density profiles allows a determination of the large-scale distribution of ionospheric conductances not only in temporal, but also in spatial resolution. The maximum Hall and Pedersen conductances are relatively high, but not unusual for the substorm expansion phase of the observations.

Acknowledgements. The Editor-in-chief thanks a referee for his help in evaluating this paper.

\section{References}

Aikio, A. T., and K. U. Kaila, A substorm observed by EISCAT and other ground-based instruments - evidence for near-earth substorm initiation, J. Atmos. Terr. Phys., 58, 1-4, 1996.

Aso, T., T. Hashimoto, M. Abe, T. Ono, and M. Ejiri, On the analysis of aurora stereo observations, J. Geomagn. Geoelectr., 42, 579-595, 1990.

Budinger, T. F., and G. T. Gullberg, Three-dimensional reconstruction in nuclear medicine emission imaging, IEEE Trans. Nucl. Sci., 21, 2-20, 1974.

Budinger, T. F., G. T. Gullberg, and R. H. Huesman, Emission computed tomography, in Image reconstruction from projections, Ed. G.T. Herman, Springer, New York, pp 147-246, 1979.

Doe, R. A., J. D. Kelly, J. L. Semeter, and D. P. Steele, Tomographic reconstruction of $630.0-\mathrm{nm}$ emission structure for a polar cap arc, Geophys. Res. Lett., 24, 1119-1122, 1997.
Foley, J. D. and A. Van Dam, Fundamentals of interactive computer graphics, Addison-Wesley, Reading, Mass., 1982.

Frey, S., H. U. Frey, D. J. Carr, O. H. Bauer, and G. Haerendel, Auroral emission profiles extracted from three-dimensional reconstructed arcs, J. Geophys. Res., 101, 21731-21741, 1996a.

Frey, H. U., S. Frey, O. H. Bauer, and G. Haerendel, Threedimensional reconstruction of the auroral arc emission from stereoscopic optical observations, SPIE Proc., 2827, 142-149, 1996b.

Frey, H. U., W. Lieb, O.H. Bauer, H. Höfner, and G. Haerendel, CCD-camera system for stereoscopic optical observations of the aurora, SPIE Proc., 2863, 460-466, 1996c.

Gilbert, P., Iterative methods for the three-dimensional reconstruction of an object from projections, J. Theor. Biol., 36, 105117, 1972.

Gordon, R., and G. T. Herman, Three-dimensional reconstruction from projections: a review of algorithms, Int. Rev. Cytol., 38, 111-151, 1974.

Gustavsson, B., A study of feasible tomographic inversion techniques for ALIS, Tech. Rep., 39, Inst. för Rymdfysik, Kiruna, Sweden, 1992.

Hedin, A. E., Extension of the MSIS thermosphere model into the middle and lower atmosphere, J. Geophys. Res., 96, 1159-1172, 1991.

Jakowski, N., E. Sardon, E. Engler, A. Jungstand, and D. Klähn, Relationship between GPS-signal propagation errors and EISCAT observations, Ann. Geophysicae, 14, 1429-1436, 1996.

Katsulai, H., and N. Arimizu, An iterative reconstruction from truncated projection data, IEEE Trans. Nucl. Sci., 32, 12171224, 1985.

Lanchester, B. S., J. R. Palmer, M. H. Rees, D. Lummerzheim, K. Kaila, and T. Turunen, Energy flux and characteristic energy of an elemental auroral structure, Geophys. Res. Lett., 21, 27892792, 1994.

Lester, M., J. A. Davies, and T. S. Virdi, High-latitude Hall and Pedersen conductances during substorm activity in the SUNDIAL-ATLAS campaign, J. Geophys. Res., 101, 26719-26728, 1996.

Markkanen, M., M. Lehtinen, T. Nygrén, J. Pirttilä, P. Henelius, E. Vilenius, E. D. Tereshchenko, and B. Z. Khudukon, Bayesian approach to satellite radio tomography with applications in the Scandinavian sector, Ann. Geophysicae, 13, 1277-1287, 1995.

Natterer, F., The mathematics of computerized tomography, Wiley, New York, 1986.

Nygrén, T., M. Markkanen, M. Lehtinen, E. D. Tereshchenko, B. Z. Khudukon, O. V. Evstafiev, and P. Pollari, Comparison of Fregion electron density observations by satellite radio tomography and incoherent-scatter methods, Ann. Geophysicae, 14, 1422-1428, 1996.

Omholt, A., The optical aurora, Springer, New York, 1971.

Pakula, W. A., P. F. Fougere, J. A. Klobuchar, J. J. Kuenzler, M. J. Buonsanto, J. M. Roth, J. C. Foster, and R. E. Sheehan, Tomographic reconstruction of the ionosphere over North America with comparison to ground-based radar, Radio Sci., 30, 89-103, 1995.

Peyrin, F. C., The generalized back projection theorem for cone beam reconstruction, IEEE Trans. Nucl. Sci., 32, 1512-1519, 1985.

Pryse, S. E., and L. Kersley, A preliminary experimental test of ionospheric tomography, J. Atmos. Terr. Phys., 54, 1007-1012, 1992.

Ratcliffe, J. A., An introduction to the ionosphere and magnetosphere, University Press, Cambridge, 1972.

Raymund, T. D., Comparison of several ionospheric tomography algorithms, Ann. Geophysicae, 13, 1254-1262, 1995.

Rees, M. H., Auroral ionisation and excitation by incident energetic electrons, Planet. Space Sci., 11, 1209-1218, 1963.

Röttger, J., Incoherent-scatter observations of the auroral ionosphere with the EISCAT radar facility, in Auroral physics, Eds. C.-I. Meng, M. J. Rycroft, and L. A. Frank, University Press, Cambridge, pp. 419-437, 1991. 
Schlegel, K., Auroral zone E-region conductivities during solar minimum derived from EISCAT data, Ann. Geophysicae, 6, 129-138, 1988.

Vallance Jones, A., et al., The ARIES auroral modeling campaign: characterization and modeling of an evening auroral arc observed from a rocket and a ground-based line of meridian scanners, Planet. Space Sci., 39, 1677-1705, 1991.
Verhoeven, D., Limited-data computed tomography algorithms for the physical sciences, Appl. Opt., 32, 3736-3754, 1993.

Walker, I. K., J. A. T. Heaton, L. Kersley, C. N. Mitchell, S. E. Pryse, and M. J. Williams, EISCAT verification in the development of ionospheric tomography, Ann. Geophysicae, 14, 1413-1421, 1996. 